\title{
Factors influencing adherence to the new intermittent preventive treatment of malaria in pregnancy policy in Keta District of the Volta region, Ghana
}

Alren O. Vandy ${ }^{1}$, Nana Yaw Peprah², Joseph Yaw Jerela ${ }^{3}$, Perfect Titiati ${ }^{3}$, Abubakar Manu', Joseph Akamah', Ernest T. Maya ${ }^{1}$ and Kwasi Torpey ${ }^{1 *}$ (D)

\begin{abstract}
Background: About 25\% of pregnant women in malaria-endemic areas are infected with malaria and this accounts for about $15 \%$ of maternal deaths globally. Intermittent preventive treatment in pregnancy with sulfadoxinepyrimethamine (IPTp-SP) is one of the main strategies for prevention of malaria in pregnancy. A new recommendation was made by the World Health Organization (WHO) that at least three doses of IPTp-SP should be administered before delivery. This study sought to determine the factors influencing adherence to the new IPTp-SP policy in Keta District, Volta region, Ghana.

Methods: A cross-sectional quantitative study among 375 nursing mothers at four selected health facilities in Keta district, Ghana was conducted using a structured questionnaire to interview participants. Sampling proportionate to the size of facility was used to determine the number of nursing mothers from each facility based on the caseload. For each facility systematic random sampling was used to select eligible nursing mothers. Data was analyzed using STATA 15. Chi-square was used to test bivariate association between categorical variables and adherence. Logistic regression analysis was used to examine sociodemographic, individual and institutional factors influencing adherence to IPTp-SP.

Result: About $82.1 \%$ of participants adhered to the WHO policy recommendations of at least three doses of IPTpSP. However, only $17.1 \%$ received Ghana's five dose coverage recommendation. The proportion of IPTp-SP coverage for IPTp1 was 98.9\%; IPTp2 95.5\%; IPTp3 80.8\%; IPTp4 39.5\%; IPTp5 17.1\%.

Conclusion: Adherence to IPTp-SP was satisfactory according to WHO's policy recommendation, however, majority of the participants had less than the five doses recommended in Ghana. Number of Antenatal Care (ANC) visits and knowledge of malaria were the main determinants of adherence to IPTP-SP.
\end{abstract}

Keywords: Malaria, Adherence, IPTp-SP, Volta region, Ghana

\footnotetext{
* Correspondence: ktorpey@hotmail.com

${ }^{1}$ University of Ghana College of Health Sciences, Accra, Ghana

Full list of author information is available at the end of the article
}

(c) The Author(s). 2019 Open Access This article is distributed under the terms of the Creative Commons Attribution 4.0 International License (http://creativecommons.org/licenses/by/4.0/), which permits unrestricted use, distribution, and reproduction in any medium, provided you give appropriate credit to the original author(s) and the source, provide a link to the Creative Commons license, and indicate if changes were made. The Creative Commons Public Domain Dedication waiver (http://creativecommons.org/publicdomain/zero/1.0/) applies to the data made available in this article, unless otherwise stated. 


\section{Background}

Malaria is a life-threatening disease that is caused by a parasitic protozoan, plasmodium. It is endemic in 91 countries and nearly $50 \%$ of the world's population at the start of 2016 were susceptible to malaria [1]. Majority of malaria reported deaths occurs in sub-Saharan Africa and about 212 million new cases and 429,000 deaths were reported globally [2].

In Sub Saharan Africa with at least 25\% of pregnant women infected with malaria in areas endemic for malaria and it accounts for $15 \%$ of maternal deaths globally [2].

About 25 million women in Sub Saharan Africa become pregnant each year and are at risk of malaria infection [3]. Ten thousand of these pregnant women and 200,000 of newborns die due to malaria in pregnancy [4].

Vulnerability of pregnant women to malaria is associated with hormonal and immunological changes in pregnancy [5]. First and second pregnancies are more susceptible to malaria and the level of parasitaemia decreases with increasing numbers of pregnancies [6].

Studies in Ghana have shown that malaria during pregnancy increases maternal anemia and low birth weight especially among women living in rural communities [7]. It is a major cause of morbidity and mortality with an estimated 382,862 pregnant women suffering from malaria in 2016 [8]. The Volta region in Ghana has been known to be one of the highest malaria prevalence regions in Ghana [9]. In 2017, the Volta region recorded the lowest score for 2016 Ghana Health Service National Health League Table and performed poorly in maternal health service delivery [10].

WHO recommends three main strategies for the treatment and prevention of malaria during pregnancy. These approaches are: intermittent preventive treatment in pregnancy (IPTp) with sulfadoxine-pyrimethamine (SP), using long lasting insecticidal nets (LLINs), and early diagnosis and treatment of malaria cases [11].

Prevention of malaria in pregnancy forestalls complications of severe malaria and reduces the risk to both pregnant women and their unborn child.

Chemoprophylaxis with IPTp-SP improves the maternal and neonatal outcomes and is recommended in regions where there is moderate to high transmission of malaria [12].

In 2007, the first WHO recommendation policy on IPTp-SP was made and it recommended that all pregnant women attending antenatal care (ANC) services should be given two doses of SP during pregnancy. The first dose of SP should be given at the start of the second trimester and second dose at the start of the third trimester. It should be given as directly observed treatment (DOT) during antenatal visits [12]. In Ghana, three doses of SP was given to pregnant women from 16 to 36 weeks [13]. In 2012, WHO Evidence Review Group reviewed new evidence from published and unpublished studies on IPTp with SP and agreed that more than two doses would be more effective in preventing malaria in pregnancy. A new recommendation was made that the first dose of IPTp-SP should be administered as early as possible in the second trimester and each dose of SP should be given at least 1 month apart up until delivery [14]. This update was done to maximize the number of SP doses given. However, according to WHO, about $69 \%$ of pregnant women in sub-Saharan Africa did not have access to the recommended three or more doses of IPTp-SP [11].

Ghana transitioned from the three doses to the five dose IPTp-SP recommendation in 2014 [15]. Currently, only a few studies have focused on the implementation of the new IPTp-SP policy that was adopted.

This study sought to determine the factors influencing adherence to the new IPTp-SP policy in Keta District, Volta region, Ghana.

\section{Methods \\ Study design, population and setting}

A facility-based cross-sectional quantitative study was conducted in May-June, 2018 to examine the factors influencing adherence to the new IPTp-SP policy. Participants were nursing mothers who had delivered within 3 months and attending the Child Welfare Clinics (CWC) and Postnatal Clinic at four health facilities in the Keta District of the Volta region, Ghana.

\section{Sample size calculation and sampling procedures}

A minimum sample size of 360 was computed with the Cochran (1977) formula, using an IPTp3 prevalence of $37.5 \%$ in the Volta region (NMCP 2017); 95\% confidence interval; a margin of error of $5 \%$.

Four health facilities with the highest volume of antenatal attendees and nursing mothers were purposively selected as recruitment sites out of twenty-eight facilities.

A sampling proportional to the number of nursing mothers seen per facility was used to determine the number of respondents recruited from each of the four facilities. Nursing mothers who gave written consent and had delivered within 3 months prior to data collection and visiting postnatal clinic or CWC at each selected health facilities were interviewed. Systematic random sampling was used for the selection of nursing mothers at the CWC who met the criteria. The sampling interval was determined by using estimated average clinic attendance per month and facility sample size. The first client was randomly selected by balloting and the interval applied for nursing mother attending the CWC until the sample size was reached. 


\section{Data collection}

An interviewer-administered questionnaire was used to collect information on socio-demographic characteristics of the nursing mothers, knowledge on malaria in pregnancy, knowledge on IPTp and SP doses (Additional file 1). Gestational age at first ANC visit, number of ANC visits and number of SP doses before delivery was extracted from the ANC Card. Information on health system/institutional factors were also elicited using a questionnaire administered to staff of antenatal clinic (Additional file 2). Table 1 shows the list of study variables, indicators, operational definition and scale of measurement.

\section{Measures}

\section{Outcome variables}

Primary outcome measure is the adherence to the new IPTp-SP policy in the district.

Secondary outcome measures include knowledge level of IPTp-Sp policy among pregnant women.

\section{Data management and analysis}

Data were entered and cleaned in Microsoft Excel 2016, and exported to Stata 15 for analysis.
Descriptive statistics using frequencies and percentage were used to describe the background characteristics the respondents. Variables were considered for inclusion in the multiple logistic regression model if their $p$-values were 0.05 in the bivariate analyses to control for the confounding and to determine factors independently associated with adherence to IPTp-SP. Adjusted odd ratios (AOR) and their 95\% confidence intervals were used to assess the strength of association. In all analyses, a $p$ value of 0.05 was used to determine statistical significance. Knowledge of malaria in pregnancy was measured by asking questions about malaria transmission and interventions with a total score of 13 . Scores of $0-5$ was considered poor, 5-10 fair and more than 10 was good. All analyses were performed in Stata 15 (StataCorp LLC, College Station, Texas).

\section{Results}

Socio-demographic characteristics of study participants A total of 375 nursing mothers within the reproductive age group (15-49 years) in the 4 selected health facilities were interviewed for the study. Table 2 summarizes the socio-demographic characteristics of the participants.

Table 1 List of Study Variables

\begin{tabular}{|c|c|c|c|}
\hline Variables & Indicators & Operational definition & $\begin{array}{l}\text { Scale of } \\
\text { Measurement }\end{array}$ \\
\hline Adherence to IPTp & Adherence & 3 or more doses of IPTp-SP & Binary \\
\hline \multirow[t]{7}{*}{ Sociodemographic } & Age & Age of respondents in years & Continuous \\
\hline & Marital Status & Marital status of respondents & Categorical \\
\hline & Religion & Religion of respondents & Categorical \\
\hline & Educational Level & Educational level attained & Categorical \\
\hline & Employment status & $\begin{array}{l}\text { Unemployed, Employed } \\
\text { or Self-employed }\end{array}$ & Categorical \\
\hline & Occupation & Current occupation of respondents & Categorical \\
\hline & Parity & Number of live births & Continuous \\
\hline \multirow[t]{3}{*}{$\begin{array}{l}\text { ANC Attendance and Obstetric } \\
\text { Characteristics }\end{array}$} & Number of ANC Visits & $\begin{array}{l}\text { Number of ANC visits during most } \\
\text { recent pregnancy }\end{array}$ & Binary \\
\hline & Gestational age at first ANC visit & $\begin{array}{l}\text { The age of pregnancy (fetus) in weeks } \\
\text { at which first ANC visit was made }\end{array}$ & Continuous \\
\hline & Gestational age at first IPTp-SP dose & $\begin{array}{l}\text { The age of pregnancy (fetus) in weeks } \\
\text { at which first IPTp-SP dose was received }\end{array}$ & Continuous \\
\hline Individual factors & $\begin{array}{l}\text { Knowledge level on Malaria, } \\
\text { MiP and IPTp }\end{array}$ & $\begin{array}{l}\text { Knowledge measured by answers } \\
\text { to questions }\end{array}$ & Categorical \\
\hline \multirow[t]{5}{*}{$\begin{array}{l}\text { Health System / } \\
\text { Institutional factors }\end{array}$} & SP availability & $\begin{array}{l}\text { Availability of SP at time } \\
\text { of data collection }\end{array}$ & Binary \\
\hline & Access to IPTp Services & $\begin{array}{l}\text { Provision of IPTp-SP services } \\
\text { at health facility }\end{array}$ & Binary \\
\hline & $\begin{array}{l}\text { Knowledge level of ANC Health Workers } \\
\text { on new IPTp-SP policy recommendation }\end{array}$ & $\begin{array}{l}\text { Knowledge measured by answers } \\
\text { to questions. }\end{array}$ & Categorical \\
\hline & Training of ANC Healthcare Workers & $\begin{array}{l}\text { ANC Healthcare workers who } \\
\text { had formal training on IPTp. }\end{array}$ & Binary \\
\hline & DOT practices & Practice of DOT for IPTp delivery. & Binary \\
\hline
\end{tabular}


Table 2 Sociodemographic characteristics of participants

\begin{tabular}{|c|c|c|}
\hline Variable & Frequency $(n)$ & Percentage (\%) \\
\hline \multicolumn{3}{|l|}{ Age grouping } \\
\hline$<18$ & 9 & 2.4 \\
\hline $18-25$ years & 132 & 35.2 \\
\hline $26-35$ years & 199 & 53.1 \\
\hline $36-45$ years & 35 & 9.3 \\
\hline Total & 375 & 100 \\
\hline \multicolumn{3}{|l|}{ Marital Status } \\
\hline Single & 24 & 6.4 \\
\hline Married & 344 & 91.7 \\
\hline Divorce / Separated & 3 & 0.8 \\
\hline Widowed & 4 & 1.1 \\
\hline Total & 375 & 100 \\
\hline \multicolumn{3}{|l|}{ Educational level } \\
\hline No formal education & 61 & 16.3 \\
\hline Primary & 119 & 31.7 \\
\hline Secondary & 169 & 45.1 \\
\hline Bachelor's degree & 18 & 4.8 \\
\hline Post graduate degree & 2 & 0.5 \\
\hline Others & 6 & 1.6 \\
\hline Total & 375 & 100 \\
\hline \multicolumn{3}{|l|}{ Employment Status } \\
\hline Employed & 40 & 10.7 \\
\hline Self-employed & 236 & 62.9 \\
\hline Unemployed & 99 & 26.4 \\
\hline Total & 375 & 100 \\
\hline \multicolumn{3}{|l|}{ Occupation } \\
\hline Trader & 111 & 40.1 \\
\hline Teacher & 22 & 7.9 \\
\hline Artisan & 94 & 33.9 \\
\hline Farmers / Fishmongers & 23 & 8.3 \\
\hline Healthcare Workers & 5 & 1.8 \\
\hline Others & 22 & 7.9 \\
\hline Total & 277 & 100 \\
\hline \multicolumn{3}{|l|}{ Religion } \\
\hline Christians & 331 & 88.3 \\
\hline Muslims & 14 & 3.7 \\
\hline Traditionalist & 30 & 8.0 \\
\hline Total & 375 & 100 \\
\hline \multicolumn{3}{|l|}{ Parity } \\
\hline 1 & 101 & 26.9 \\
\hline 2 & 118 & 31.5 \\
\hline$\geq 3$ & 155 & 41.3 \\
\hline Missing data & 1 & 0.3 \\
\hline Total & 375 & 100 \\
\hline
\end{tabular}

In all, 53.1\% (199/375) of the participants were between the ages of $26-35$ years and the majority $(91.7 \%, 344 / 375)$ were married with $41.3 \%$ (15/375) having three or more children. Nearly half of them had secondary level education $45.1 \%,(169 / 375)$ and most of the participants $(62.9 \%$, 236/375) were self-employed as traders and artisans.

Majority of the participants $88.1 \%(331 / 375)$ were Christians followed by traditionalist $(8.0 \%, 30 / 375)$ and then Muslims $(3.7 \%, 14 / 375)$ (Table 2).

\section{ANC attendance and obstetric characteristics of participants}

Table 3 presents descriptive information on ANC attendance and obstetrics characteristics of the nursing mothers. About two-thirds of the nursing mothers $(66.1 \%, 248 / 375)$

Table 3 ANC and Obstetric Characteristics of participants

\begin{tabular}{|c|c|c|}
\hline Variable & Frequency $(n)$ & Percentage (\%) \\
\hline \multicolumn{3}{|c|}{ Gestational age at first ANC } \\
\hline$\leq 13$ weeks & 165 & 44.0 \\
\hline 13-26 weeks & 199 & 53.1 \\
\hline$\geq 26$ weeks & 11 & 2.9 \\
\hline Total & 375 & 100 \\
\hline \multicolumn{3}{|c|}{ Number of ANC visits } \\
\hline$<8$ & 248 & 66.1 \\
\hline$\geq 8$ & 127 & 33.9 \\
\hline Total & 375 & 100 \\
\hline \multicolumn{3}{|c|}{ Gestational age at first IPTp-SP } \\
\hline$\leq 13$ weeks & 11 & 3.0 \\
\hline $13-26$ weeks & 339 & 90.4 \\
\hline$\geq 26$ weeks & 21 & 5.6 \\
\hline Don't know & 4 & 1.1 \\
\hline Total & 375 & 100 \\
\hline \multicolumn{3}{|c|}{ Number of IPTp-SP doses } \\
\hline 0 & 4 & 1.1 \\
\hline 1 & 13 & 3.5 \\
\hline 2 & 50 & 13.3 \\
\hline 3 & 160 & 42.7 \\
\hline 4 & 84 & 22.4 \\
\hline$\geq 5$ & 64 & 17.1 \\
\hline Total & 375 & 100 \\
\hline
\end{tabular}

Number of SP tablet given per dose

$\begin{array}{lll}\leq 2 & 29 & 7.7 \\ 3 & 330 & 88.0 \\ >3 & 12 & 3.2 \\ \text { Don't know } & 4 & 1.1 \\ \text { Total } & 375 & 100\end{array}$

$n$ number of respondents, ANC antenatal care, IPTp-SP Intermittent Preventive Treatment in Pregnancy with sulphadoxine pyrimethamine, $S P$ sulphadoxine pyrimethamine 
had less than the eight WHO recommended ANC visits/ contacts during pregnancy, 33.9\% (127/375) had eight or more ANC visits during the period of pregnancy. The mean number of ANC was $6.5 \pm 2.6$ visits.

More than half $(53.1 \%, 199 / 375)$ of the participants had their first ANC visit during the second trimester (13-26 weeks), 44.0\% (165/375) during the first trimester and only $2.9 \%(11 / 375)$ had their first ANC visit in the third trimester. The mean gestational age at first ANC visit was $14.4 \pm 6.5$ weeks.

In all, $90.4 \%(339 / 375)$ of the respondents had their first dose of IPTp-Sp during the second trimester between 13 and 26 weeks of gestation and 21 (5.7\%) respondents had theirs in the third trimester of pregnancy.

Thirteen respondents $(3.5 \%$; 13/375) had one dose of IPTp-SP with the majority $(42.7 \%, 160 / 375)$ having three doses of IPTp-SP before delivery. About 17.1\% (64/375) had five doses or more of IPTp-SP before delivery. Only four respondents $(1.1 \%)$ did not take SP during their pregnancy. Some respondents reported side effects and allergies after taking SP tablets.

When asked the number of SP tablets taken per dose, $(88.0 \%, 330 / 375)$ reported of receiving three tablets of SP per dose, 29 (7.7\%) reported of receiving less than three SP tablets and $12(3.2 \%)$ mentioned more than three SP tablets per dose (Table 3).

\section{Adherence to new IPTp-SP policy recommendations and proportion of IPTp-SP coverage}

Majority of the participants $(82.1 \%, 308 / 375)$ had three or more doses of IPTp-SP during their pregnancy as recommended by WHO and only $17.9 \%(67 / 375)$ had less than three doses.
However, when using Ghana's five dose IPTp-SP coverage recommendation, only $64(17.1 \%)$ participants adhered.

The proportion of IPTp-SP coverage for IPTp1 was 98.9\%; IPTp2 95.5\%; IPTp3 82.1\% IPTp4 39.5\%; IPTp5 17.1\% (Fig. 1).

\section{Individual factors}

Over half of the respondents (52.0\%) had a fair knowledge about malaria and Malaria in Pregnancy (MiP), $42.8 \%$ had poor knowledge and only $5.2 \%$ had good knowledge about malaria and MiP. Majority $(83.5 \%, 308 / 375)$ of respondents had knowledge about the IPTp-SP and the benefits of it. The main source of information on malaria was from ANC/Health facility followed by the media.

\section{Healthcare system/institutional factors}

All the selected health facilities offered IPTp services (both static and outreach services).

SP was the drug of choice at these facilities; however, one out of the four facilities did not have SP at the time the study was conducted but was available few months prior to data collection.

SP was given as DOT at all the health facilities where the study was conducted and clean drinking water (sachets) was available for pregnant women. Of the 11 staff interviewed, six (54.6\%) of respondents have had training on IPT-SP.

Assessment of Knowledge of ANC staff on IPTp showed that $90.9 \%$ of the ANC workers knew the correct definition of IPTp. All respondents (100.0\%) knew the recommended drug, the dose and the correct interval for IPTp. However, only $45.5 \%$ knew when to start IPTp and $81.8 \%$ knew when it was contraindicated during pregnancy. This is shown in Table 4.

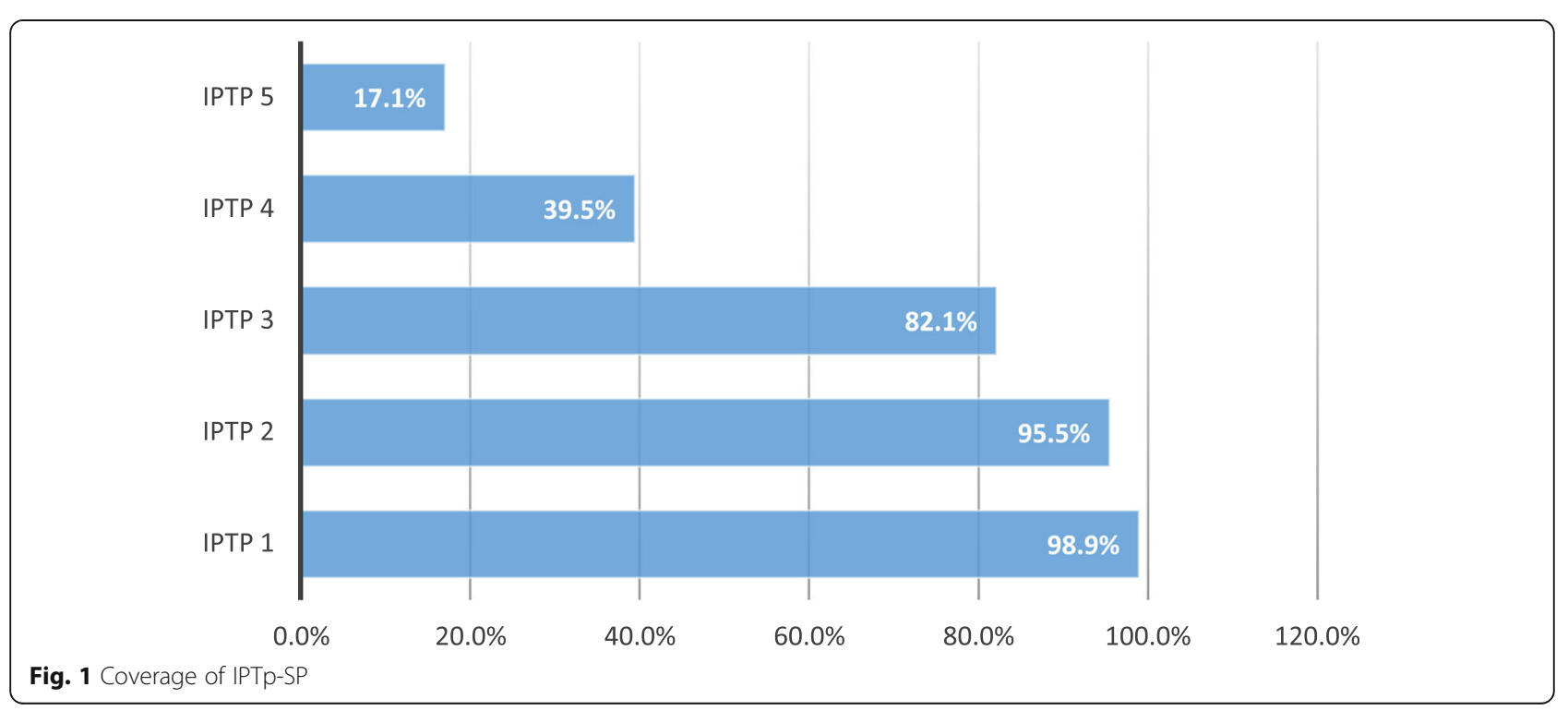


Table 4 Knowledge of ANC Workers on IPTp-SP

\begin{tabular}{lll}
\hline Knowledge $N=11$ & Frequency & Percentage (\%) \\
\hline Correct definition of IPTp & 10 & 90.9 \\
Recommended drug for IPTp in Ghana & 11 & 100.0 \\
When to start IPTp & 5 & 45.5 \\
When not to give IPTp & 9 & 81.8 \\
Recommended dose for IPTp in Ghana & 11 & 100.0 \\
Correct interval for IPTp & 11 & 100.0 \\
\hline
\end{tabular}

Factors associated with adherence to IPTp-SP

In the multivariate logistic regression model, after adjusting for characteristics of participants, having $\geq 8$ $\mathrm{ANC}$ visits $(\mathrm{AOR}=4.51,95 \%$ CI 1.76-11.57, $p<0.05)$ and knowledge of IPTp-SP $(\mathrm{AOR}=2.74,95 \% \mathrm{CI} 1.29-$ $5.82, p<0.05)$ were significantly associated to adherence to IPTp-SP. This is shown in Table 5.

\section{Discussion}

According to National Malaria Control Programme, IPTp-SP coverage for Volta region in 2017 was IPTp 163.7\%, IPTp 2-53.0\%, IPTp 3-37.5\%, IPTp 4-18.8\%, IPT $5-5.9 \%$ which appears to be lower than those found in the present study [16]. However, in the same year, a study conducted in the Accra Metropolitan area in Ghana had similar findings to the current study and reported IPTp1 to be 98.8\%, IPTp 2-94.9\%, IPTp3-87.5\%, IPTp 4-55.7\%, IPTp 5-14.5\% [17].

In a study by Sikambale, Halwindi, \& Baboo (2013) in Zambia, IPTp3 coverage was found to be much lower (30\%). About a third (28.8\%) had no IPTp-SP dose taken during their most recent pregnancy [18]. In our study, only $1.1 \%$ of the respondents did not have any dose of IPTp-SP and IPTp 3 coverage was much higher.

Several studies have shown an increase in adherence to IPTp-SP due to ongoing campaigns and increase in coverage. According to WHO (2016), there is an increase in adherence to the IPTp-SP policy in malariaendemic regions and at least $50 \%$ of women reported to have received one or more doses [2]. Also, only $19 \%$ of eligible pregnant women had three or more doses of IPTp-SP compared to $18 \%$ in the previous year [2].

A study conducted in Mali on uptake of IPTp-SP found a higher uptake of SP of three doses more than the Demographic and Health Survey reported data [19]. Nonetheless, Mpogoro et al. (2014) in Tanzania identified a much lower proportion of pregnant women adhering to IPTpSP compared to the national survey report [20].

The number of ANC visits was the main determinant of adherence to IPTp-SP in the current study though gestational age at first ANC and dose was significant. Most of the previous studies focused on the antenatal care model of four ANC visits during pregnancy which
Table 5 Multivariate analysis of factors associated with adherence

\begin{tabular}{|c|c|c|c|}
\hline & $\mathrm{AOR}$ & $95 \% \mathrm{Cl}$ & $\mathrm{p}$ \\
\hline \multicolumn{4}{|l|}{ Age grouping } \\
\hline$<18$ & Ref & & \\
\hline $18-25$ & 2.46 & $0.43-14.13$ & 0.311 \\
\hline $26-35$ & 5.50 & $0.94-32.08$ & 0.058 \\
\hline $36-45$ & 4.49 & $0.58-34.81$ & 0.151 \\
\hline \multicolumn{4}{|l|}{ Religion } \\
\hline Christian & Ref & & \\
\hline Muslim & 1.00 & & \\
\hline Traditionalist & 0.52 & $0.19-1.42$ & 0.201 \\
\hline \multicolumn{4}{|l|}{ Educational level } \\
\hline No formal education & Ref & & \\
\hline Primary & 0.86 & $0.36-2.05$ & 0.734 \\
\hline Secondary and above & 2.21 & $0.84-5.84$ & 0.109 \\
\hline \multicolumn{4}{|l|}{ Gestational age at first ANC } \\
\hline$\leq 13$ weeks & Ref & & \\
\hline 13-26 weeks & 1.22 & $0.60-2.49$ & 0.589 \\
\hline$>26$ weeks & 0.68 & $0.09-4.93$ & 0.705 \\
\hline \multicolumn{4}{|c|}{ Gestational age at first IPTp-SP } \\
\hline$\leq 13$ weeks & Ref & & \\
\hline $13-26$ & 0.94 & $0.17-5.35$ & 0.946 \\
\hline$>26$ & 0.15 & $0.02-1.37$ & 0.093 \\
\hline \multicolumn{4}{|l|}{ Number of ANC visits } \\
\hline$<8$ & Ref & & \\
\hline$>=8$ & 4.51 & $1.76-11.57$ & $0.002^{*}$ \\
\hline \multicolumn{4}{|l|}{ Knowledge on Malaria } \\
\hline Poor & Ref & & \\
\hline Average & 1.34 & $0.67-2.67$ & 0.402 \\
\hline Good & 3.26 & $0.30-35.21$ & 0.33 \\
\hline \multicolumn{4}{|l|}{ Knowledge of IPTp-SP } \\
\hline No knowledge & Ref & & \\
\hline Knowledge & 2.74 & $1.29-5.82$ & 0.009 \\
\hline
\end{tabular}

AOR adjusted odds ratio, $95 \% \mathrm{Cl} 95 \%$ confidence interval, Ref reference *- $p<0.05$

also showed significant association with adherence to IPTp-SP.

ANC visits promote uptake of IPTp-SP prevents malaria in pregnancy, protects mother and the unborn baby from complications of malaria in pregnancy and improves perinatal outcome [12, 21].

Early detection of pregnancy is important for early commencement of ANC [22]. In Ghana, there is a government policy of free maternal care and most ANC services are covered by the national health insurance scheme [23]. A study in Cameroon showed that there was a significant 
association between amount of SP doses taken and early first ANC attendance (at an early gestation age). Pregnant women who had early first ANC attendance were more likely to receive the recommended doses of SP [24].

Various studies have been conducted to assess the relationship between knowledge and IPTp-SP use. Studies in Zambia and Nigeria have shown that knowledge level of pregnant women on IPTp-SP strongly influences their adherence to the IPTp-SP recommendations and women were 2.6 times more likely to complete IPTp-SP doses $[18,25]$. The studies also support the finding from the current study that knowledge on malaria and IPTp-SP use is average among pregnant women. This can be associated with the level of education of the respondents from the study.

To reiterate, education empowers women with knowledge which in turn enables women to make informed choices and in this case, choice of ANC and IPTp-SP use.

Institutional factors such as knowledge of the health care workers and their capacity is essential to operationalize the policy direction into public health practice. While the knowledge on IPTp was generally good, most of the healthcare workers had difficulties on when to start prophylaxis. This is particularly important as any uncertainties on when to start is likely to delay the initiation of IPTp and limit the ability to deliver five doses before delivery. Enhancing staff capacity is an important element to improve uptake and early initiation of IPTp [26].

The study was well powered and provides useful insight in the implementation of the new policy in Keta District. However, it cannot be generalized to represent the entire Volta region or Ghana. In addition, there may be recall bias from participants as the interviews took place a few months after delivery.

\section{Conclusions}

Adherence to IPTp- SP in the study was determined to be $82.1 \%$ with WHO's recommendation of 3 or more doses of IPTp and $17.1 \%$ with Ghana's 5 dose policy recommendation.

Number of ANC visits during pregnancy was one of the main determinants of adherence to IPTp-SP. Majority $(66.1 \%)$ of the participants had less than the recommended 8 ANC visit during pregnancy.

Health care workers had a good knowledge of IPTp but were less certain on when to start the prophylaxis.

\section{Supplementary information}

Supplementary information accompanies this paper at https://doi.org/10 1186/s12884-019-2544-8.

Additional file 1. Questionnaire for nursing mothers

Additional file 2. Questionnaire for staff for antenatal clinic

\section{Abbreviation}

ANC: Antenatal Care; AOR: Adjusted Odd Ratios; Cl: Confidence Interval; CWC: Child Welfare Clinics; DOT: Directly Observed Treatment; IPTp: Intermittent Preventive Treatment in Pregnancy; IPTp-SP: Intermittent Preventive Treatment in Pregnancy with Sulfadoxine-Pyrimethamine; MiP: Malaria in Pregnancy; SP: Sulfadoxine-Pyrimethamine; WHO: World Health Organization

\section{Acknowledgements}

We would like to the National Malaria Control Programme, Keta Municipal Health Directorate, the hospital management and staff of Keta Municipal Hospital, Sacred Heart (Keta) Hospital, Tegbi Health Centre, Anloga Health Centre, research assistants and clients for their support during the study.

\section{Availability of data materials}

The datasets used and analyzed in this study are available from the corresponding author upon request.

\section{Authors' contributions}

AOV and KT conceptualized the study. AOV, NYP, JYJ, PT, AM and KT oversaw data collection. AOV analyzed the data, AOV, NYP, JYJ, PT, AM, JA, ETM and KT drafted the manuscript. AOV, NYP, JYJ, PT, AM, JA, ETM and KT provided critical input in the development and revision of the manuscript. All authors read and approved the final manuscript.

\section{Funding}

This work has been funded by TDR, the Special Programme for Research and Training in Tropical Diseases, which is hosted at the World Health

Organization and co-sponsored by UNICEF, UNDP, the World Bank and WHO to the University of Ghana (Grant \# B40300).

The views expressed in this article are those of the authors and not the funding agency. The funder of the study had no role in study design, data collection, data analysis, data interpretation, or writing of the report.

\section{Ethics approval and consent to participate}

Ethical approval number GHS-ERC:035/12/17 was granted by the Ghana Health Service Ethics Review Committee. Informed written consent was obtained from all participants after the study objectives, procedures and potential risk and benefits had been thoroughly explained. In addition, permission was obtained from the Keta District Health Directorate as well as all the four participating health facilities.

\section{Consent for publication}

Personal identifiers were excluded from the data set before analyses were performed. We therefore did not report any individual identifier.

\section{Competing interests}

The authors declare that they have no competing interests.

\section{Author details}

'University of Ghana College of Health Sciences, Accra, Ghana. ${ }^{2}$ National Malaria Control Programme, Ghana Health Service, Accra, Ghana. ${ }^{3}$ Keta Municipal Health Directorate, Keta, Ghana.

Received: 11 June 2019 Accepted: 30 September 2019 Published online: 20 November 2019

\section{References}

1. World Health Organization. Malaria [Internet]. World Malaria Report, 2017. Available from: https://www.who.int/news-room/fact-sheets/detail/malaria

2. World Malaria Report 2016. Geneva: World Health Organization. World Malaria Report. World Health Organization. 2016. 1-186 p.

3. Desai M, Kuile FO, Nosten F, Mcgready R, Asamoa K, Brabin B, et al. Epidemiology and burden of malaria in pregnancy. 2007;7(February).

4. Roman E, Ngindu A, Orji B, Zoungrana J, Robbins S, Brieger W. International Journal of Gynecology and Obstetrics Evolution of malaria in pregnancy control : Jhpiego 's 10-year contribution. Int J Gynecol Obstet [Internet] 2015;130:S62-7. Available from: https://doi.org/10.1016/j.ijgo.2015.03.009

5. Robinsona DP, Kleina SL. Pregnancy and pregnancy-associated hormones alter immune responses and disease pathogenesis. Horm Behav. 2013;62(3):263-71. 
6. Stephens JK, Kyei-Baafour E, Dickson EK, Ofori JK, Ofori MF, Wilson ML, et al. Effect of IPTp on Plasmodium falciparum antibody levels among pregnant women and their babies in a sub-urban coastal area in Ghana. Malar J [Internet]. 2017;16(1):224. Available from: http://malariajournal. biomedcentral.com/articles/10.1186/s12936-017-1857-1

7. Ofori M, Ansah E, Agyepong I, Ofori-Adjei D, Hviid L, Akanmori B. Pregnancy-associated malaria in a rural community of Ghana. Ghana Med J. 2009:43(1):13-8.

8. 8th Issue of Ghana National Malaria Control Programme (NMCP) Peroidic Bulletin [Internet]. 2016 [cited 2019 Jun 9]. Available from: https:// ghanahealthservice.org/downloads/2016 -Annual_Bulletin.pdf

9. Prevalence and Management of Malaria in Ghana: A Case Study of Volta Region Chuks J. Mba 1 and Irene K. Aboh 2.

10. GHANA HEALTH SERVICE. GHANA HEALTH SERVICE 2016 Annu Rep 2017.

11. WHO. World Malaria Day 2017. 2017;(April):1-5.

12. WHO. WHO policy brief for the Implementation of Intermittent Preventive Treatment of Malaria in Pregnancy Using Sulfadoxine-Pyrimethamine (IPTpSP). WHO Press. 2014;(October 2012):1-13.

13. PRESIDENT ' S MALARIA INITIATIVE Ghana Malaria Operational Plan FY 2014. 2014;

14. Updated WHO Policy Recommendation. 2012 [cited 2017 Sep 15]; Available from: http://www.who.int/malaria/iptp_sp_updated_policy_ recommendation_en_102012.pdf

15. NMCP. President's Malaria Initiative, Ghana. Malaria Operational Plan FY 2017. Cdc. 2016;9-11.

16. NMCP Programme Data. Ghana National Malaria Control Programme (NMCP) IPTp Coverage. August 2017:2017.

17. Boateng IO, Anto F. Intermittent preventive treatment of malaria in pregnancy : a cross - sectional survey to assess uptake of the new sulfadoxine - pyrimethamine five dose policy in Ghana. Malar J. 2017:1-9.

18. Sikambale C, Halwindi H, Baboo KS. Factors influencing utilization of intermittent presumptive treatment of malaria (\{IPTp\}) services by pregnant women in \{Sesheke\} district of \{Western\} \{province\} \{Zambia\}. Med J Zambia. 2013;40(1):24-32.

19. Hurley EA, Harvey SA, Rao N, Diarra NH. Underreporting and Missed Opportunities for Uptake of Intermittent Preventative Treatment of Malaria in Pregnancy ( IPTp ) in Mali. 2016;1-17.

20. Mpogoro FJ, Matovelo D, Dosani A, Ngallaba S, Mugono M, Mazigo HD. Uptake of intermittent preventive treatment with sulphadoxinepyrimethamine for malaria during pregnancy and pregnancy outcomes : a cross-sectional study in Geita district,. 2014;1-14.

21. Nkoka O, Chuang TW, Chen YH. Association between timing and number of antenatal care visits on uptake of intermittent preventive treatment for malaria during pregnancy among Malawian women. Malar J [internet]. 2018;17(1):1-11. Available from: https://doi.org/10.1186/s12936-018-2360-z.

22. Morroni $C$, Moodley J. The role of urine pregnancy testing in facilitating access to antenatal care and abortion services in South Africa: a crosssectional study. BMC Pregnancy Childbirth. 2006;6:1-7.

23. Gobah FK, Zhang L. The National Health Insurance Scheme in Ghana: prospects and challenges: a cross-sectional evidence. Glob J Health Sci. 2011:3(2):90-101.

24. Anchang-Kimbi JK, Achidi EA, Apinjoh TO, Mugri RN, Chi H, Tata RB, et al. Antenatal care visit attendance, intermittent preventive treatment during pregnancy (IPTp) and malaria parasitaemia at delivery. Malar J [Internet]. 2014;13(1):162. Available from: http://malariajournal.biomedcentral.com/ articles/10.1186/1475-2875-13-162

25. Akinleye SO, Falade CO, Ajayi IO. Knowledge and utilization of intermittent preventive treatment for malaria among pregnant women attending antenatal clinics in primary health care centers in rural southwest, Nigeria: a cross-sectional study. BMC Pregnancy Childbirth. 2009;9(1):28.

26. Arulogun OS, Okereke CC. Knowledge and practices of intermittent preventive treatment of malaria in pregnancy among health workers in a southwest local government area of Nigeria. J Med Med Sci [Internet] 2012; 3(6):415-22. Available from: http://www.interesjournals.org/JMMS

\section{Publisher's Note}

Springer Nature remains neutral with regard to jurisdictional claims in published maps and institutional affiliations.

\section{Ready to submit your research? Choose BMC and benefit from:}

- fast, convenient online submission

- thorough peer review by experienced researchers in your field

- rapid publication on acceptance

- support for research data, including large and complex data types

- gold Open Access which fosters wider collaboration and increased citations

- maximum visibility for your research: over $100 \mathrm{M}$ website views per year

At BMC, research is always in progress.

Learn more biomedcentral.com/submissions 